\title{
A High Aspect Ratio 2D Gimbaled Microscanner with Large Static Rotation
}

\author{
Sunghoon Kwon, Veljko Milanovic, ${ }^{\dagger}$ Luke P. Lee \\ 1plee@socrates.berkeley.edu
}

\author{
Berkeley Sensor and Actuator Center \\ Department of Bioengineering \\ University of California, Berkeley, CA 94720
}

\author{
${ }^{\dagger}$ Adriatic Research Institute \\ 2131 University Avenue Suite 322 \\ Berkeley, CA 94704-1029
}

\begin{abstract}
We introduce a simple isolation method for SOI MEMS technologies. The proposed isolation method by backside island process provides electrical isolation and mechanical coupling of SOI structures by timed deep reactive ion etching (DRIE) of backside substrate. Adding the backside island to our previously demonstrated vertical comb drives, we have fabricated SOI-based 2D gimbaled microscanners with large static rotation. The fabricated devices perform large static optical deflection from $-20.3^{\circ}$ to $15.6^{\circ}$ by outer gimbal and and from $0^{\circ}$ to $-11.9^{\circ}$ by inner mirror.
\end{abstract}

\section{INTRODUCTION}

Silicon-on-insulator (SOI) micromirror provides attractive features such as flat, reflective, and robust device layer, etch stop, CMOS compatibility, and simple fabrication for MOEMS applications [1]. Staggered mirror performs well for both static and dynamic application by taking these advantages [2]. However, it is limited to 1degree of freedom (DoF) uni-directional rotation due to the electrically coupled lower combfingers. Recently, we have developed independently and linearly controllable vertical comb drives using SOI [3]. In addition to the controllability, its advantages include large displacement and bi-directional actuation. Our goal was to expend these advantages to $2 \mathrm{DoF}$ application.

Gimbaled structure is most common and effective way of implementing 2DoF rotation. Hybrid 2DoF mirror has been demonstrated by gap closing actuation, which requires additional bonding and linearization [5]. To implement 2DoF gimbaled micromirror without cross talk between driving voltages, electrical isolation and mechanical coupling are necessary. Backfilling of isolation trench by additional deposition layer and following chemical mechanical polishing (CMP) has been used to achieve the electrically isolated mechanical coupling [4]. However, the additional deposition and CMP steps debase the advantage of using SOI.

In this paper, we present SOI-based 2DoF gimbaled micromirror with large static rotation by backside island isolation process. The proposed isolation method not only eliminates the need for backfilling and linearization but also enable SOI

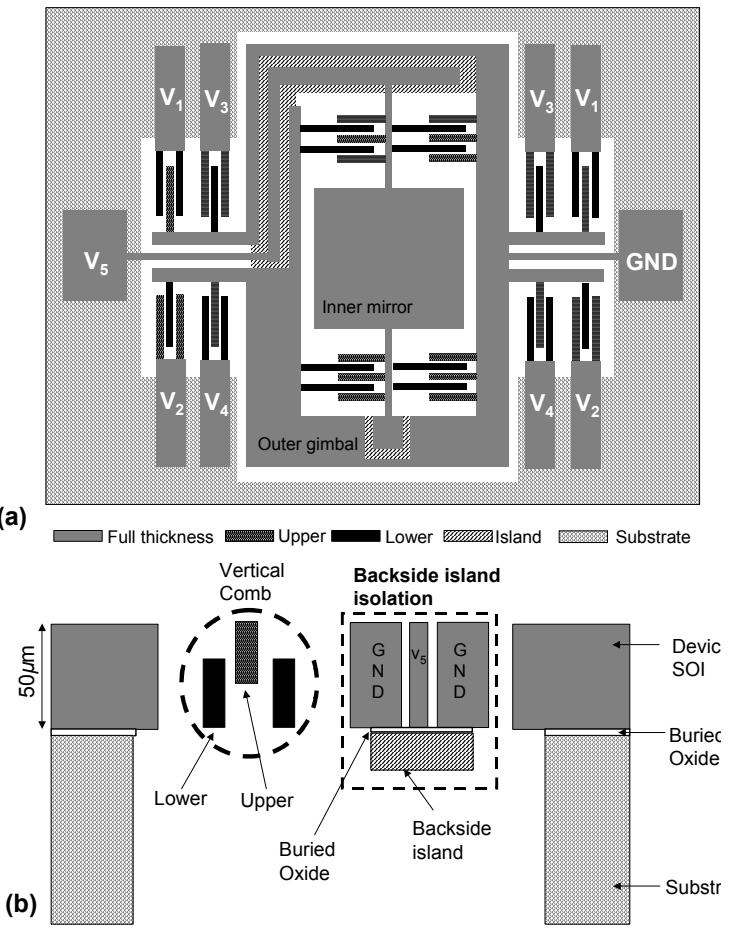

Figure 1. (a) Top view of design of $2 \mathrm{DoF}$ gimbaled micromirror denoted by beams in different level. V denotes driving voltage. Different pattern means different crossection. (b) Cross sectional view of required beams. Note the backside island is connecting two full SOI beams with different voltage.

gimbaled structure only by adding one more patterning step to previously developed vertical comb drive.

\section{DESIGN AND FABRICATION}

The 2D gimbaled mirror is designed using 5 different silicon beams with different thickness. The cross section of the required beams is shown in Fig. 1a. The design (Fig. 1) utilizes a two-axis gimbal, where inner axis is electrically isolated with outer gimbal whereas they are mechanically coupled. This isolation and coupling is done by a timed-etched backside $\mathrm{Si}$ island below the gimbal structure as shown in dashed rectangle of Fig.1b. This island together with isolated comb banks allow both upward and downward actuation of outer gimbal. Five different voltages (V1-V5) and both upward and downward actuation of outer gimbal. 

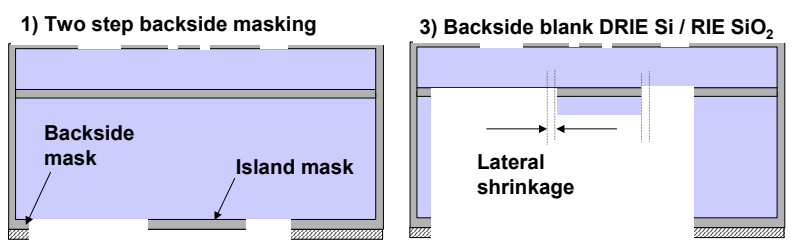

2) Backside DRIE Si/ RIE $\mathrm{SiO}_{2}$

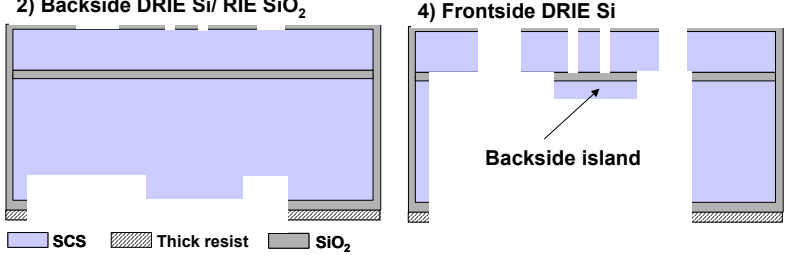

Figure 2. Backside island process for isolation. Substrate is timed etched from the back to form island.

The fabrication process for backside island is shown in Fig. 2. The fabrication process for vertical comb drive is not shown here and can be found in [3]. The thin backside island is formed by two step DRIE etching from the backside. (1) After masks are patterned on oxide and thick photoresist layer, (2) timed DRIE from the back is performed to set the thickness of island. Remained island mask is cleaned by RIE. (3) Blank DRIE from the back transfer the step height for island until buried oxide is exposed. Lateral shrinkage about $10 \%$ should be considered in design due to the blank etching. Buried oxide is cleaned by RIE. The buried oxide under the island work as insulated isolated connector for the top device structure. (4) Finally, the device structure is defined from the font DRIE.

\section{RESULT}

The SEM of the fabricated device is shown in Fig. 3. The dashed box in Fig.3a is where the island is attached to the bottom of the outer gimbal. Fig. $3 b$ is the picture of island from the backside. Vertical comb with torsion spring is shown in Fig. 3c.

The demonstrated static optical deflection from gimbal rotation (tipping) from $-20.3^{\circ}$ to $0^{\circ}$ at $<100 \mathrm{~V}$ on pads $\mathrm{V} 1 \& \mathrm{~V} 2$, and from $0^{\circ}$ to $15.6^{\circ}$ for $<100 \mathrm{~V}$ on pads $\mathrm{V} 3 \& \mathrm{~V} 4$. Inner axis (tilting) from $0^{\circ}$ to $-11.9^{\circ}$ was measured for $<100 \mathrm{~V}$ on pad V5. Pistoning up was performed by actuating $\mathrm{V} 1 \& \mathrm{~V} 3$, and pistoning down by actuating pads V2\&V4, giving static pistoning from of $13 \mu \mathrm{m}$ p-to-p at $<100 \mathrm{~V}$. Table 1 shows the summary of the preliminary result on optical deflection.

\section{CONCLUSION}

2DoF microscanner with large static deflection is demonstrated by combination of vertical comb drive and the proposed backside island isolation.

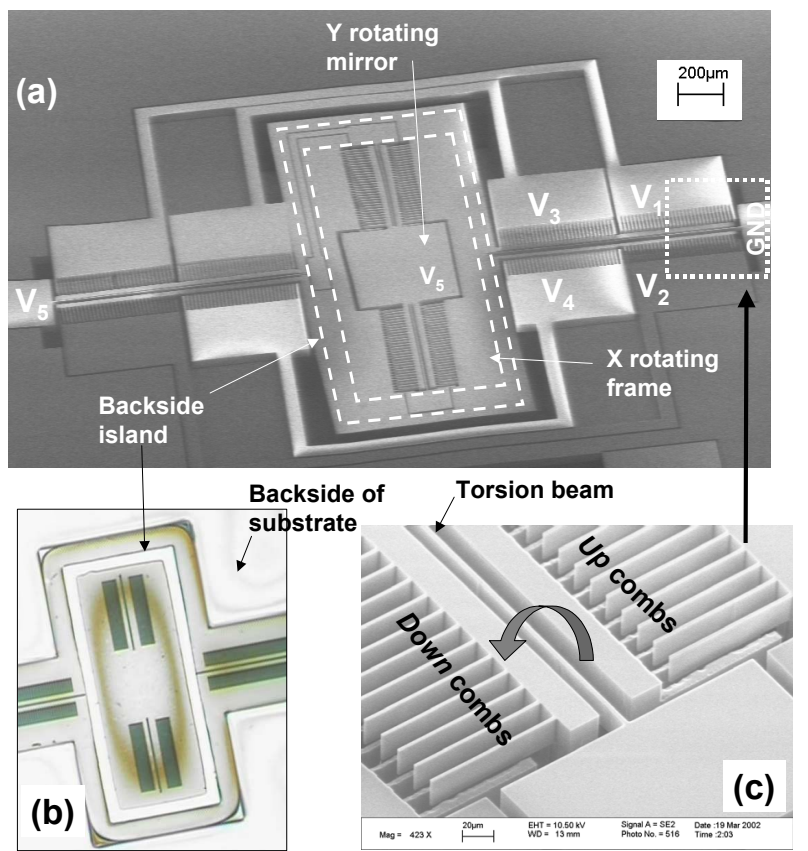

Figure 3. (a) The SEM of $2 \mathrm{DoF}$ gimbaled micromirror using SOI (Topview), (b) The microscope picture of backside island (Bottom view), and (c) Vertical combdrives with torsion beam

Successful electrical isolation as well as mechanical coupling can be achieved.

\begin{tabular}{|c|c|c|c|c|c|}
\hline \multicolumn{7}{|c|}{ 2DoF Micromirror } \\
\hline Outer axis [opt. deflect.] & \multicolumn{2}{|c|}{ Inner axis [opt. deflect.] } & \multicolumn{2}{c|}{ Pistoning $[\mu \mathrm{m}]$} \\
\hline+ & - & + & - & + & - \\
\hline $7.0^{\circ}$ at $50 \mathrm{~V}$ & -12.0 at $50 \mathrm{~V}$ & $7.6^{\circ}$ at $50 \mathrm{~V}$ & $\mathrm{n} / \mathrm{a}$ & $\mathrm{n} / \mathrm{a}$ & $\mathrm{n} / \mathrm{a}$ \\
\hline $15.6^{\circ}$ at $100 \mathrm{~V}$ & $-20.3^{\circ}$ at $100 \mathrm{~V}$ & $11.9^{\circ}$ at $100 \mathrm{~V}$ & $\mathrm{n} / \mathrm{a}$ & 7.0 at $100 \mathrm{~V}$ & -6.0 at $100 \mathrm{~V}$ \\
\hline
\end{tabular}

Table 1. Static optical deflection of 2DoF mirror

\section{REFERENCES}

[1] S. Blackstone and T. Brosnihan, "SOI MEMS technologies for Optical Switching, " 2001 IEEE/LEOS International Conference on Optical MEMS, pp.35-36, 2001

[2] Conant, R, Nee, J, Lau, KY, Muller, RS, “A Flat High-Frequency Scanning Micromirror," Proc. SolidState Sensor and Actuator Workshop, Hilton Head, South Carolina, pp. 6-9, June 4-8, 2000.

[3] S. Kwon, V. Milanovic, and L. P. Lee, "Vertical microlens scanner for 3D Imaging," Proc. Solid-State Sensor and Actuator Workshop, Hilton Head, South Carolina, 2002.

[4] H. Schenk, P. Durr, D. Kunze, H. Lakner, and H. Kuck, "A resonantly excited 2D-micro-scanning-mirror with large deflection," Sensors and Actuators A, vol 89, pp.104-111, 2001

[5] G. J. Su, H. Toshiyoshi, and M. C. Wu, "SurfaceMicromachined 2-D Optical Scanners with HighPerformance Single-Crystalline Silicon, " IEEE Photonics Technology Letters, Vol.13, No. 6, 2001 